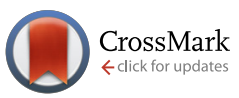

Cite this: Med. Chem. Commun., $2015,6,1444$

Received 13th March 2015,

Accepted 8th June 2015

DOI: $10.1039 / \mathrm{c} 5 \mathrm{md} 00108 \mathrm{k}$

www.rsc.org/medchemcomm

\title{
Effect of dibucaine hydrochloride on raft-like lipid domains in model membrane systems $\dagger$
}

\begin{abstract}
Kazunari Yoshida, ${ }^{* a}$ Akito Takashima ${ }^{b}$ and Izumi Nishio ${ }^{b}$
To clarify the biophysical and/or physicochemical mechanism of anaesthesia, we investigated the influence of dibucaine hydrochloride (DC.HCl), a local anaesthetic, on raft-like domains in ternary liposomes composed of dioleoylphosphatidylcholine (DOPC), dipalmitoylphosphatidylcholine (DPPC) and cholesterol (Chol). The large DOPC/DPPC/Chol liposome is directly observable by optical microscopy and is known to be laterally separated into a liquid-ordered (Lo) phase (raft-like domain) and liquid-disordered (Ld) phase under certain conditions. Hence, it is useful for the study of lipid raft domains as a simple model system of cell membranes. We observed ternary liposomes with three concentrations $(0,0.05$ and $0.2 \mathrm{mM})$ of $\mathrm{DC} \cdot \mathrm{HCl}$ at various temperatures and calculated the angle-averaged two-dimensional autocorrelation functions to confirm the change in miscibility transition temperature $\left(T_{c}\right)$ of ternary liposomes. Furthermore, we calculated the circularity of the Lo domain to confirm the change in line tension of the Lo/Ld phase boundary. The results indicated that the insertion of DC molecules into lipid bilayers induces reduction in $T_{c}$ of the ternary liposome, accompanied by reduction in line tension of the phase boundary. This suggests that the $\mathrm{DC} \cdot \mathrm{HCl}$ molecules may disturb ion channel functions by affecting the lipid bilayers surrounding the ion channels.
\end{abstract}

\section{Introduction}

Understanding the biophysical and/or physicochemical mechanisms of the functional expression of anaethestics in pharmacology is important. As such, the effect of anaesthetic molecules on cell membranes containing ion channels has been extensively studied. For instance, the effect of chloroform and other general anaethestics on the sodium current in a squid giant axon and interactions of chloroform and/or benzyl alcohol with the lipid bilayer have been studied through experimental and/or simulation methods. ${ }^{1-5}$

Recently, local anaethestics have attracted significant attention. Numerous studies have been reported involving the effect of local anaethestics on the sodium current across the cell membrane. ${ }^{6-12}$ In addition, interactions between anaethestics and the lipid bilayer, which surrounds the ion channels, have also been studied as it has been suggested that the perturbation of the lipids due to invasion of anaethestics disturbs the ion channel function. ${ }^{13-17}$

\footnotetext{
${ }^{a}$ New Industry Creation Hatchery Center, Tohoku University, 6-6-10 Aoba, Aramaki, Aoba-ku, Sendai 980-8579, Japan. E-mail: kazunari.yoshida.a3@tohoku.ac.jp ${ }^{b}$ Department of Physics and Mathematics, College of Science and Engineering, Aoyama Gakuin University, 5-10-1 Fuchinobe, Chuo-ku, Sagamihara, Kanagawa 252-5258, Japan

$\dagger$ Electronic supplementary information (ESI) available: Typical results of the 2D-AC analyses of liposomes with three types of phase patterns, a typical microscopic image of an ambiguous phase pattern, and result of Fourier analysis. See DOI: $10.1039 / \mathrm{c} 5 \mathrm{md} 00108 \mathrm{k}$
}

Furthermore, it is still unknown how local anaethestics affect lipid raft domains. Lipid raft domains are mainly composed of saturated lipids, cholesterol and membrane proteins, such as ion channels, and are dispersed in cell membranes, which are thought to occur via phase separation. ${ }^{18,19}$ Since lipid rafts play an important role in various cell functions, ${ }^{18}$ the effect of local anaethestics on raft domains should be investigated to clarify their detailed mechanism of action. Phaseseparated liposomes with raft-like domains have been used as simple model systems of the cell membrane to study the interactions between local anaethestics and lipid bilayers. ${ }^{20,21}$ However, the detailed physicochemical mechanism of the functional expression of local anaesthesia is still unclear.

Large phase-separated liposomes that are observable by optical microscopy are useful for the direct investigation of raft-like domains. Multicomponent liposomes are known to be separated into two or three phases due to the difference in thermodynamic properties of their lipids. In addition, the phase-separated domains of large liposomes are observable by fluorescence microscopy with a dye incorporated in a certain phase. ${ }^{22-27}$ Indeed, large phase-separated liposomes have been used as models for many studies, revealing the properties of the lipid membrane. ${ }^{22,24-30}$

In cases similar to the present study, the large liposome and cell-derived giant plasma membrane vesicle (GPMV), which is similar to the multicomponent liposome, are also used as model systems. For example, it has been reported that vitamin E, Triton-X 100 and benzyl alcohol affect the 
phase morphology of large multicomponent liposomes. ${ }^{31}$ Gray et al. reported that general anaethestics, including alcohols, reduce the miscibility transition temperature $\left(T_{\mathrm{c}}\right)$ of GPMVs. ${ }^{32}$ They estimated the percentage of phase-separated vesicles and determined $T_{\mathrm{c}}$ as the temperature where $50 \%$ of vesicles have two phases. However, it is difficult to distinguish one- or two-phase liposomes near the critical point using this method as the phase boundary becomes unclear. In other words, determination of a threshold between oneand two-phase liposomes is very difficult near the critical point.

In the present study, we investigated the influence of dibucaine hydrochloride (DC·HCl), a commonly used local anaesthetic, on the phase behaviour of liposomes composed of dioleoylphosphatidylcholine (DOPC), dipalmitoylphosphatidylcholine (DPPC) and cholesterol (Chol). The DOPC/DPPC/ Chol liposome is a system known to be laterally separated into liquid-ordered (Lo) and liquid-disordered (Ld) phases under certain conditions. ${ }^{23}$ The Lo phase, mainly composed of DPPC and Chol, represents the raft-like domain, while the Ld phase represents the DOPC-rich region. ${ }^{23}$ We calculated angle-averaged two-dimensional autocorrelation (2D-AC) functions of images of liposomes with three concentrations of $\mathrm{DC} \cdot \mathrm{HCl}$ at various temperatures without determining a threshold of one- and two-phase liposomes to quantify the phase pattern of liposomes and estimate the change in $T_{\mathrm{c}}$. The results indicated that DC molecules reduce the $T_{\mathrm{c}}$ of ternary liposomes.

The 2D-AC and angle-averaged 2D-AC functions have been widely used in various fields, such as biology ${ }^{33}$ and physics, ${ }^{34,35}$ to quantify the image pattern, such as dotted patterns. $^{34,35}$ In addition, angle-averaged 2D-AC analysis was performed to clarify the correlation length of the GPMV surface in a similar study. ${ }^{36}$ To the best our knowledge, this is the first report demonstrating the change in $T_{\mathrm{c}}$ of ternary liposomes with addition of local anaethestics by calculating the angle-averaged 2D-AC function of membrane domain patterns.

\section{Materials and methods}

\subsection{Materials}

$\mathrm{DC} \cdot \mathrm{HCl}$, DOPC (chain melting temperature, $T_{\mathrm{m}} \approx-18{ }^{\circ} \mathrm{C}$ ), ${ }^{37}$ DPPC $\left(T_{\mathrm{m}} \approx-41^{\circ} \mathrm{C}\right),{ }^{37} \mathrm{Chol}$ and chloroform were purchased

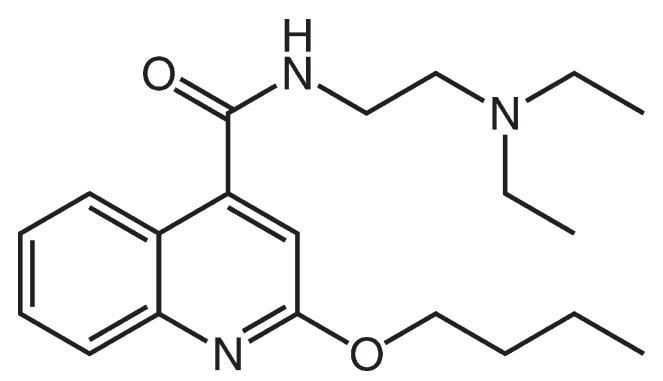

Fig. 1 Chemical structure of dibucaine (DC). from Wako Pure Chemical Industries, Ltd. (Japan). The chemical structure of DC is shown in Fig. 1. Methanol was purchased from Showa Chemical Industry Co., Ltd. (Japan). Rhodamine B 1,2-dihexadecanoyl-sn-glycero-3-phosphoethanolamine, triethylammonium salt (rhodamine DHPE, $\lambda_{\mathrm{ex}}=560 \mathrm{~nm}$, $\lambda_{\mathrm{em}}=580 \mathrm{~nm}$ ), a fluorescent phospholipid, was obtained from Invitrogen (USA). Ultra-pure water was obtained using a WT101UV Autopure system (Yamato Scientific Co., Ltd., Japan).

\subsection{Preparation of ternary liposomes}

We prepared liposomes using natural swelling methods. ${ }^{38,39}$ The lipids of DOPC/DPPC/Chol = 50/25/25 (mol\%) and rhodamine DHPE were dissolved in a solvent mixture of chloroform and methanol with a volume ratio of $2: 1$. The organic solvents were then removed using an air flow and placing the sample in an aspirated desiccator for more than $8 \mathrm{~h}$ to form a dry lipid film. Finally, liposomes were formed through hydration of the lipid film with ultra-pure water at $37{ }^{\circ} \mathrm{C}$ for more than $24 \mathrm{~h}$. The lipids DOPC/DPPC/Chol and rhodamine DHPE had concentrations of $0.2 \mathrm{mM}$ and $1 \mu \mathrm{M}$, respectively.

\subsection{Observations}

The liposome suspension $(40 \mu \mathrm{L})$ and aqueous $\mathrm{DC} \cdot \mathrm{HCl}$ solution $(40 \mu \mathrm{L})$ were gently mixed, and the solution was incubated at room temperature (RT, $21.7 \pm 0.4^{\circ} \mathrm{C}$ ) for more than 10 min to achieve sufficient dispersion of $\mathrm{DC} \cdot \mathrm{HCl}$ molecules into the lipid membrane. In this study, we used $0,0.1$ and $0.4 \mathrm{mM}$ of aqueous DC.HCl solutions. The final DOPC/DPPC/ Chol concentration was $0.1 \mathrm{mM}$ with $0,0.05$ or $0.2 \mathrm{mM}$ $\mathrm{DC} \cdot \mathrm{HCl}$ and $0.5 \mu \mathrm{M}$ rhodamine DHPE. The mixed solution (5 $\mu \mathrm{L}$ ) was then placed between two cover glasses and sealed with vacuum grease. The sample was placed on a copper plate, and the temperature of the plate was controlled using a Peltier device (deviation $<0.1{ }^{\circ} \mathrm{C}$ ) and monitored using a thermistor probe placed in the copper plate (under the sample). A thin layer of thermal grease was placed between the sample cell and copper plate to maintain high thermal conduction from the plate to the sample. The sample placed on the plate was incubated for more than $10 \mathrm{~min}$ to achieve thermal equilibrium prior to observation. The sample was then observed using a fluorescence microscope BX40 (Olympus, Japan) through a $40 \times$ objective lens at various temperatures. The microscopic images were recorded using a DP73 digital camera (Olympus, Japan). Excitation-light irradiation $\left(\lambda_{\max }=546 \mathrm{~nm}\right)$ was applied using a $\mathrm{Hg}$ lamp through a WIG filter set (Olympus, Japan). The observed liposomes were unilamellar vesicles with a diameter range of approximately 15-40 $\mu \mathrm{m}$. The diameter of the majority of observed liposomes was $15-25 \mu \mathrm{m}$, and that of a few liposomes was nearly $40 \mu \mathrm{m}$.

\subsection{Analysis of microscopic images}

We calculated the angle-averaged 2D-AC using a macro program of ImageJ. ${ }^{40}$ First, fluorescence microscopic images of the liposome surface were cut into $64 \times 64$ pixels and converted to a greyscale (16 bit). This pixel size is appropriate 
for calculating angle-averaged 2D-AC of liposomes with a diameter greater than $15 \mu \mathrm{m}$. The influence of pixel size on $2 \mathrm{D}$-AC values is believed to be very small as most of the observed liposomes are 15-25 $\mu \mathrm{m}$. We then calculated the two-dimensional fast Fourier transform (2D-FFT) using the brightness of each pixel in the images and inverse transformed their power spectra to derive the $2 \mathrm{D}$-AC function as

$$
g(r, \theta)=\mathscr{F}^{-1}[\tilde{F}[I] \cdot \mathscr{F} *[I]],
$$

where $g(r, \theta)$ is the 2D-AC as a function of real space position $(r, \theta), \mathscr{F}$ represents 2D-FFT, $\mathscr{F}^{*}$ represents complex conjugation of the Fourier component, $\mathscr{F}^{-1}$ represents the inverse 2D-FFT and $I$ is the brightness of each pixel in the microscopic image. $\mathscr{F}[I]$ represents the $2 \mathrm{D}-\mathrm{FFT}$ of $I$. The $2 \mathrm{D}-\mathrm{AC}$ function was averaged for all angles as

$$
G(r)=\langle g(r, \theta)\rangle_{\theta},
$$

where $G(r)$ is the angle-averaged $2 \mathrm{D}$-AC as a function of radial position $(r)$ in real space. Finally, we calculated the FFT of normalised $G(r) / G(0)$ curves, which represent the correlation between the brightness of a certain point $(r=0)$ and another one placed within the radial distance $(r)$. Typical results of the analysis of three domain patterns are described in the ESI $\dagger$ (Fig. S1). It is not necessary to determine the phase pattern (one- or two-phase) of an ambiguous case such as that shown in Fig. S2.†

\subsection{Calculation of Lo-domain circularity}

Circularity was also calculated using ImageJ. ${ }^{40}$ We transformed the microscopic images to binary images to obtain the phase boundary of Lo/Ld. The circularity, a dimensionless parameter, of the Lo domains was defined as

$$
(\text { circularity })=\frac{4 \pi A}{l^{2}},
$$

where $\pi$ is the ratio of the circumference of a circle to its diameter, $A$ is the area of a Lo domain, and $l$ is the perimeter of the same Lo domain. A circularity of 1 corresponds to a perfect circle. We calculated the circularity to estimate the change in line tension of the phase boundary in the case of $T$ $=20^{\circ} \mathrm{C}$.

\section{Results and discussion}

We observed the DOPC/DPPC/Chol liposomes with $0,0.05$ and $0.2 \mathrm{mM} \mathrm{DC} \cdot \mathrm{HCl}$ at $T=20,25,30,35$ and $40{ }^{\circ} \mathrm{C}$ to confirm the influence of $\mathrm{DC} \cdot \mathrm{HCl}$ on the phase behaviour of ternary liposomes. Fig. 2 shows the typical microscopic images under each set of conditions. Since rhodamine DHPE is mainly localised in the Ld phase of phase-separated systems, ${ }^{26,41}$ the dark regions correspond to DPPC- and Cholrich domains (Lo, raft-like domain), while the bright regions correspond to DOPC-rich domains (Ld). In addition, it was previously reported that dye lipids influence the phase behaviours of ternary liposomes. ${ }^{42}$ However, rhodamine DHPE was also incorporated into control systems; hence the effect of only local anaesthetics was observed in this study. Image (1) in Fig. 2 shows the phase-separated liposome with scattered Lo domains, which disappeared with increasing temperature (images (1)-(5)). In the case of $0 \mathrm{mM}$ at $35{ }^{\circ} \mathrm{C}$, we observed many one-phase liposomes. No phase-separated liposome exists at $40{ }^{\circ} \mathrm{C}$, as shown in image (5). Veatch et al. reported

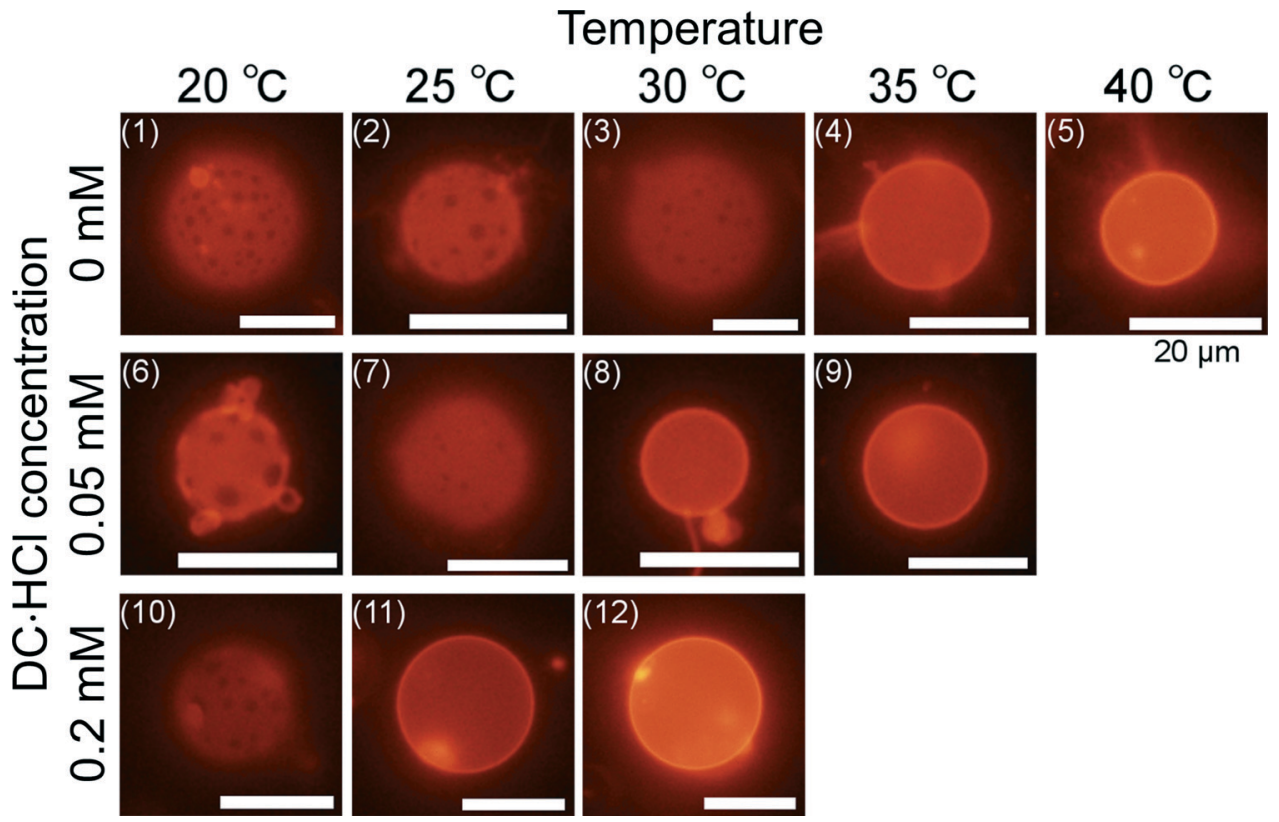

Fig. 2 Typical microscopic images of DOPC/DPPC/Chol liposomes with 0, 0.05 and $0.2 \mathrm{mM} \mathrm{DC} \cdot \mathrm{HCl}$ at 20, 25, 30, 35 and $40{ }^{\circ} \mathrm{C}$. Scale bars represent $20 \mu \mathrm{m}$. 
$T_{\mathrm{c}}=30^{\circ} \mathrm{C}$ in the case of the DOPC/DPPC $/ \mathrm{Chol}=50 / 25 / 25$ system, ${ }^{23}$ which is in agreement with the behaviour of the present system. Next, we observed the ternary liposomes at various temperatures in the case of $0.05 \mathrm{mM} \mathrm{DC} \cdot \mathrm{HCl}$. We observed phase separation in most liposomes with $0.05 \mathrm{mM}$ $\mathrm{DC} \cdot \mathrm{HCl}$ at $20^{\circ} \mathrm{C}$, as shown in image (6). There were several phase-separated liposomes with smaller Lo domains, as shown in image (7), and a few liposomes with one large domain, as shown in Fig. S1(c), $\dagger$ at $25^{\circ} \mathrm{C}$. We observed many one-phase liposomes at $30^{\circ} \mathrm{C}$ (image (8)) and $35{ }^{\circ} \mathrm{C}$ (image (9)). In the case of $0.2 \mathrm{mM}$, the number of phase-separated liposomes decreased as the temperature increased. At $20{ }^{\circ} \mathrm{C}$, the majority of liposomes were phase-separated; however, there were several liposomes which had unclear phase boundaries. At $25^{\circ} \mathrm{C}$ and above, most liposomes had one phase without raft-like domains, as shown in images (11) and (12). We focused on the images of all three $\mathrm{DC} \cdot \mathrm{HCl}$ concentrations at $25^{\circ} \mathrm{C}$, as shown in images (2), (7) and (11). The Lo domains in the liposome become smaller as the $\mathrm{DC} \cdot \mathrm{HCl}$ concentration increased. These results indicate that $\mathrm{DC} \cdot \mathrm{HCl}$ molecules have the ability to change the phase behaviour of DOPC/DPPC/Chol liposomes. Moreover, we could not confirm the influence of vesicle size on phase behaviors.

We calculated the 2D-AC functions of the ternary liposome domain pattern to quantify the phase behaviour and averaged the functions. The typical results of this analysis are shown in Fig. S1, $\dagger$ as described above. The near region from the reference point, $r=0(r \leq 0.5 \mu \mathrm{m})$, is a high correlation domain. The nearest similar phase domains generally exist at the high correlation region of $2.0 \leq r \leq 3.0$, due to phase separation, as in the case of the two-phase image with scattered Lo domains (Fig. S1(b) $\dagger$ ). Therefore, the $G(r) / G(0)$ curve of phase-separated liposomes shows a certain fluctuation due to the scattered domain pattern (Fig. S1(d) $\dagger$ ), ${ }^{34,35}$ and the Fourier amplitudes at low wavenumber (especially $k \approx 0.3$ and $0.6 \mu^{-1}$ ) have higher values (Fig. S1(e) $\dagger$ ). Furthermore, in the case of large phase separation, as shown in Fig. S1(c), $\dagger$ the high correlation region is quite long due to its large Lo or Ld domain (Fig. S1(d) $\dagger$ ) and the $G(r) / G(0)$ does not show fluctuation within $3.5 \mu \mathrm{m}$. Hence, the correlation of the $r \leq 0.5$ $\mu \mathrm{m}$ region is also very high, while the Fourier amplitude is low (Fig. S1(e) $\dagger$ ). In terms of this consideration, we must focus on both the short distance region of $G(r) / G(0)$ and the Fourier amplitudes at low wavenumber. In previous methods, the ambiguity of the phase pattern, as shown in Fig. S2, $\uparrow$ was problematic, as there was no circular domain but slight fluctuations in the lipid density. However, this method enabled us to estimate the approximate percentage of phaseseparated liposomes without determination of the one- and two-phase threshold of each liposome.

Fig. 3(a, c and e) show the mean values of $G(r) / G(0)$ as a function of radial position $(r)$ at each concentration, while Fig. 3(b, d and f) show the enlarged view of the short distance region of the angle-averaged $2 \mathrm{D}$-AC functions (mean \pm standard error), corresponding to Fig. 3(a, c and e), respectively. The error bars can be seen only in the enlarged view as they confuse the measured values in Fig. 3(a, c, and e). The number of observed liposomes was greater than 10 under each set of conditions. We focused on the correlation of the short distance region, as described above. The value of the short distance region (especially $r \approx 0.25 \mu \mathrm{m}$ ) of $G(r) / G(0)$ decreased as the temperature increased (Fig. 3(a and b)). It was determined that the correlation of the short distance region depends on the ratio of the phase-separated liposomes, because the correlation of the short distance region of the two-phase liposome is different from that of the one-phase liposome, as described above. The results in Fig. 3(b) indicate that the ratio of phase-separated liposomes decreased as the temperature increased. The difference in correlation values of $r \approx 0.25 \mu \mathrm{m}$ at several temperatures gradually decreased as the $\mathrm{DC} \cdot \mathrm{HCl}$ concentration increased. This behaviour was especially shown above $30^{\circ} \mathrm{C}$. This indicates that the percentage of phase-separated liposomes becomes similar due to the $\mathrm{DC} \cdot \mathrm{HCl}$ molecules. In other words, the percentage of phaseseparated liposomes at 20 and $25{ }^{\circ} \mathrm{C}$ decreased upon the addition of DC.HCl. It was suggested that the DC.HCl molecules disturb the phase separation of ternary liposomes. Further observations are required to clarify the detailed behavior of autocorrelations.

Since we need further information to confirm the effect of DC. HCl molecules on miscibility transition temperature $\left(T_{\mathrm{c}}\right)$, the FFT of the $G(r) / G(0)$ curves of individual liposomes was calculated and averaged. As shown in Fig. S1, $\dagger$ FFT calculations clarify the difference between one-phase (without pattern) and scattered small Lo domain patterns. Fig. S3(a-c) $\dagger$ show the Fourier spectrum of the $G(r) / G(0)$ curves at $0,0.05$ and $0.2 \mathrm{mM}$, respectively ( $n \geq 10$ ). The Fourier amplitude of phase-separated liposomes at wavenumber $k \approx 0.3$ and 0.6 $\mu^{-1}$ tends to have larger values than that of one-phase liposomes, due to Lo domains, as shown in the Fig. S1(e); $\uparrow$ the wavenumbers $k \approx 0.3$ and $0.6 \mu \mathrm{m}^{-1}$ correspond to wavelengths $\lambda \approx 3.3$ and $1.7 \mu \mathrm{m}$, respectively. Therefore, the averaged values of the Fourier amplitudes depend on the ratio of phaseseparated liposomes. The amplitudes at $k \approx 0.3$ and $0.6 \mu \mathrm{m}^{-1}$ decreased as the temperature increased at all concentrations.

The relationship between the Fourier amplitude and the temperature is shown in Fig. 4. The drop of the Fourier amplitudes with increasing temperature between 30 and 35 ${ }^{\circ} \mathrm{C}$ is shown at $0 \mathrm{mM}$, while the drop between 20 and $25^{\circ} \mathrm{C}$ is shown at 0.05 and $0.2 \mathrm{mM}$. In other words, the dropping temperature region decreased with addition of the $\mathrm{DC} \cdot \mathrm{HCl}$ molecules. This indicates that the $\mathrm{DC} \cdot \mathrm{HCl}$ molecules reduce the $T_{\mathrm{c}}$ as the dropping region roughly corresponds to $T_{\mathrm{c}}$. The $T_{\mathrm{c}}$ shifts downward more than $5{ }^{\circ} \mathrm{C}$ from $0 \mathrm{mM}$ to 0.05 and $0.2 \mathrm{mM}$. The behaviour seen in Fig. 4 differs from the results of the 2D-AC functions. This is because there are large phaseseparated liposomes, such as those seen in Fig. S1(c), $\dagger$ in the case of $0.05 \mathrm{mM} \mathrm{DC} \cdot \mathrm{HCl}$ at $25^{\circ} \mathrm{C}$. The Fourier amplitude was low in this case, while the correlation value of the short distance region was very high (Fig. S1(d and e) $\dagger$ ). This shows that both the angle-averaged 2D-AC functions and their Fourier amplitudes are required to clarify the effect of 
(a)

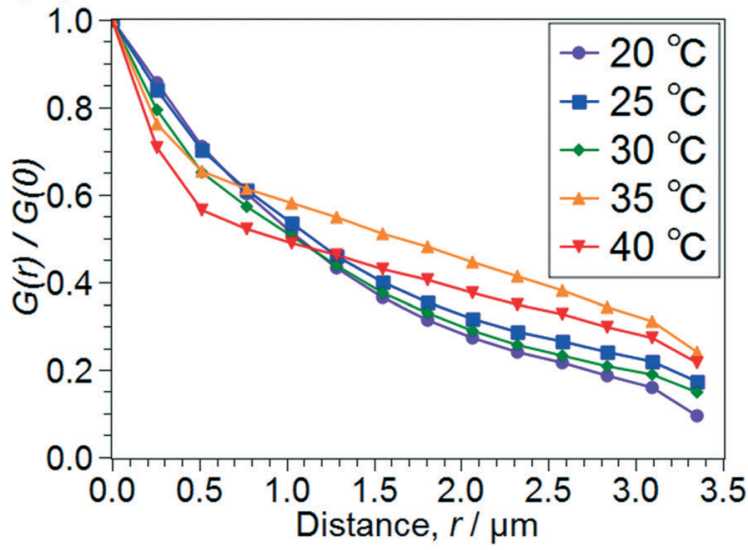

(c)

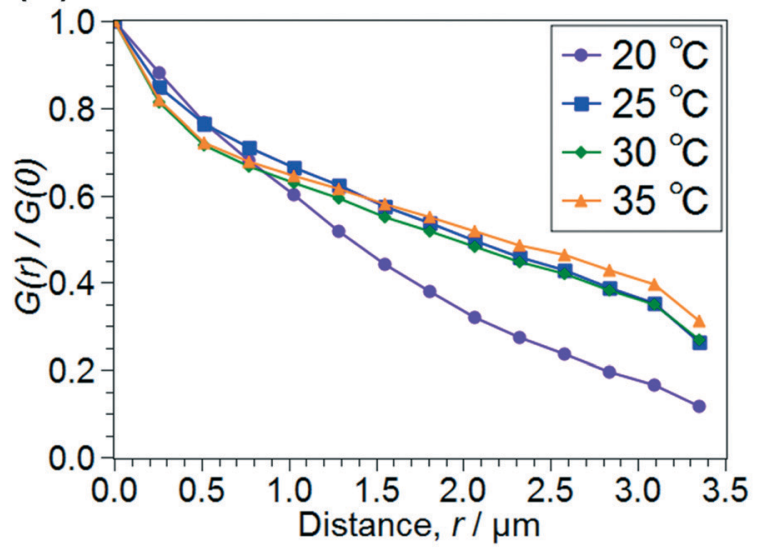

(e)

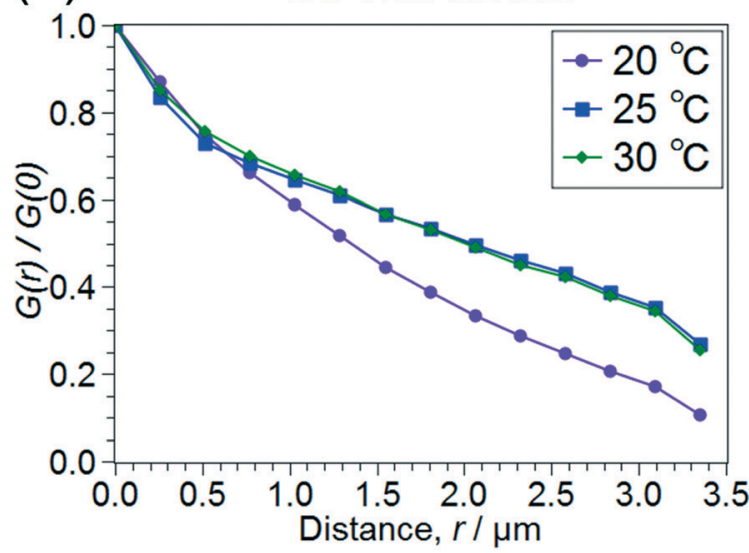

(b)

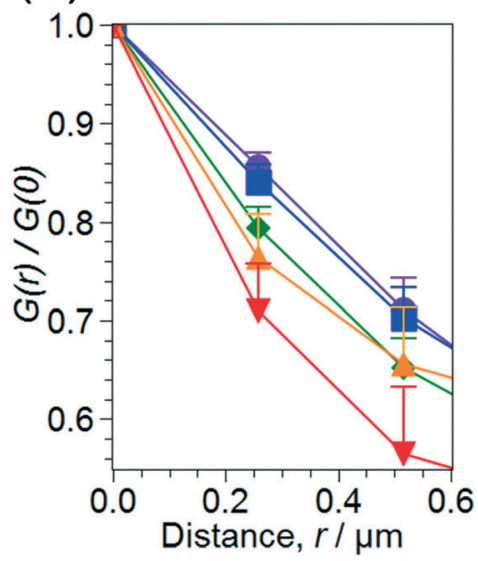

(d)

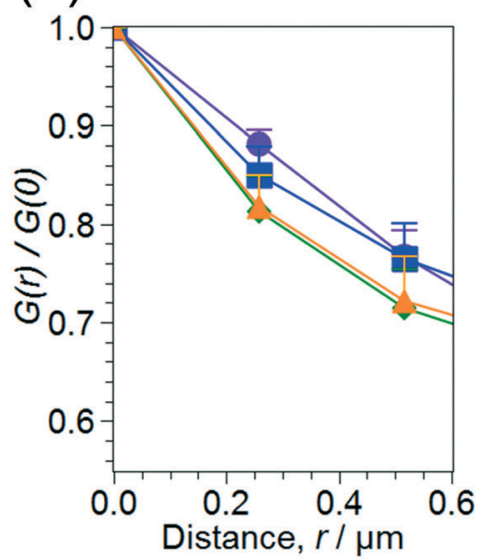

(f)

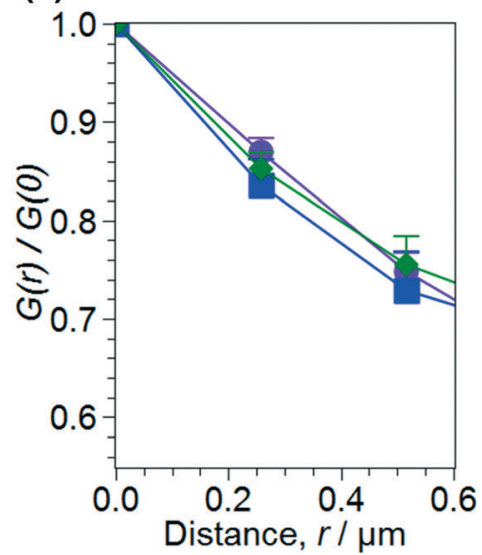

Fig. 3 Mean values of the angle-averaged 2D-AC functions ( $n \geq 10$ under each set of conditions). (a, c and e) The angle-averaged 2D-AC functions of ternary liposomes with $0,0.05$ and $0.2 \mathrm{mM} \mathrm{DC} \cdot \mathrm{HCl}$, respectively (error bars not shown). (b, $d$ and f) Enlarged view of the short distance region of the angle-averaged 2D-AC function, corresponding to $\mathrm{a}, \mathrm{c}$ and e, respectively. Error bars in enlarged view represent standard errors.

anaethestics on the phase behaviour of ternary liposomes. These results suggest that $\mathrm{DC} \cdot \mathrm{HCl}$ has the ability to decrease the $T_{\mathrm{c}}$ of ternary liposomes.

In this study, we demonstrated that $\mathrm{DC} \cdot \mathrm{HCl}$ molecules induce a decrease in $T_{\mathrm{c}}$ of DOPC/DPPC/Chol liposomes. Furthermore, we calculated the Lo domain circularity to discuss the change in the line tension of phase boundary. We were not able to determine the exact value of the line tension of the phase boundaries, as it is difficult to determine the fluctuations in Lo/Ld phase boundaries of small Lo domains. However, we could easily calculate the small Lo domain circularity, showing that the domain circularity is related to the 

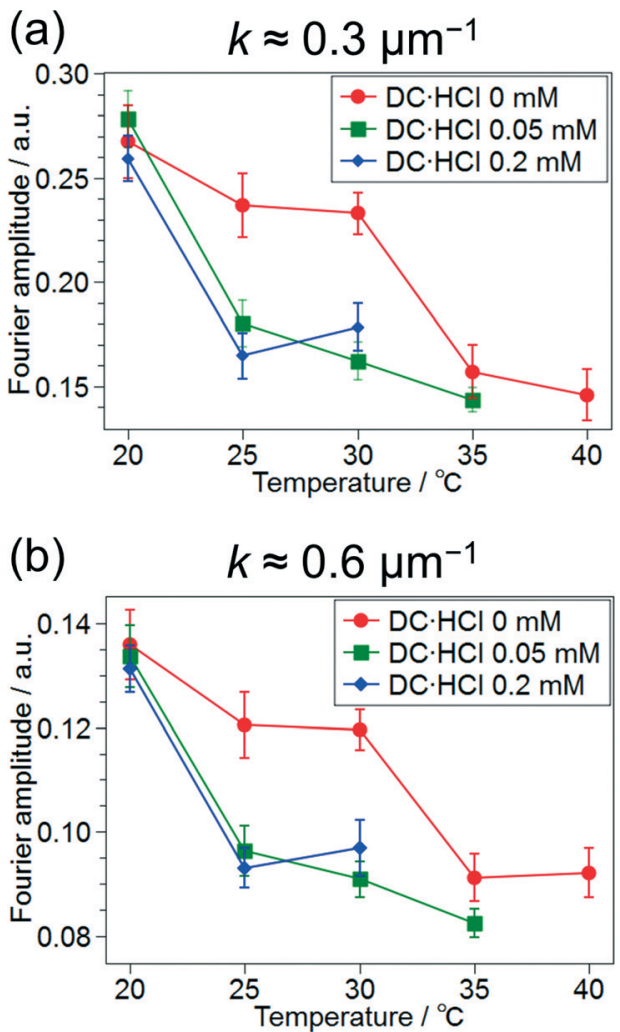

Fig. 4 ( $\mathrm{a}$ and $\mathrm{b}$ ) The Fourier amplitude versus temperature at $k \approx 0.3$ and $0.6 \mu \mathrm{m}^{-1}$, respectively. Error bars represent standard errors $(n \geq 10$ under each set of conditions).

line tension of the phase boundary, as higher line tension tends to induce more circular domains and minimises energy. ${ }^{43}$ Therefore, this is an appropriate method for these conditions, allowing us to estimate the change in line tension. Fig. 3 shows the circularity $\left(4 \pi A / l^{2}\right)$ at $20^{\circ} \mathrm{C}$. The circularity decreased as the $\mathrm{DC} \cdot \mathrm{HCl}$ concentration increased, suggesting that the line tension of the Lo/Ld phase boundary decreased with $\mathrm{DC} \cdot \mathrm{HCl}$ concentration. In other words, the $\mathrm{DC} \cdot \mathrm{HCl}$ molecules induce reduction in line tension of the phase boundary.

Near the critical point, line tension of the phase boundary was expressed as

$$
\sigma \propto\left(T_{\mathrm{c}}-T\right)^{v}
$$

where the $\sigma$ is the line tension of the Lo/Ld phase boundary and $v$ is the critical exponent. ${ }^{22,44}$ In terms of this equation, the line tension $(\sigma)$ decreased the miscibility transition temperature $\left(T_{\mathrm{c}}\right)$ when the temperature of the system $(T)$ was constant. It was determined that insertion of the DC molecules into bilayers induced the reduction in $T_{\mathrm{c}}$ (Fig. 4), leading to the decrease in line tension. DC molecules have previously been shown to have the ability to invade lipid bilayers, ${ }^{45}$ which led us to consider this possibility in the case of DOPC/DPPC/Chol systems. In addition, the change in line tension induced by the incorporation of other molecules was reported by several groups. ${ }^{46,47}$ Therefore, it was determined that the insertion of the DC molecules into bilayers induces reduction in the miscibility transition temperature and line tension of the phase boundary.

The drop of the domain circularity with increasing $\mathrm{DC} \cdot \mathrm{HCl}$ concentration between 0.05 and $0.2 \mathrm{mM}$ is shown in Fig. 5, while the difference of the Fourier amplitude between 0.05 and $0.2 \mathrm{mM}$ (Fig. 4) is very small. This is because the exact line tension of the phase boundary was not obtained, but the domain circularity was calculated. We are not able to quantitatively compare the circularity with the behaviours of the Fourier amplitudes but are able to compare the line tension with $T_{\mathrm{c}}$ using eqn (4). However, the approximate behaviour of the line tension with $T_{\mathrm{c}}$ was confirmed.

Similar studies to the present work suggested that general anaethestics disturb the phase separation of liposomes and GPMVs (reduction in $T_{\mathrm{c}}$ ) using neutron diffraction and/or X-ray diffraction methods ${ }^{48,49}$ and direct observation. ${ }^{32}$ It is believed that the behaviour shown in these previous studies was also induced by insertion of anaesthetic molecules into bilayers. In addition, Hamada et al. have reported that the photoisomerisation of an azobenzene derivative reversibly switches the one-/two-phase pattern due to the change in $T_{\mathrm{c}}$, accompanied by change in the lateral line tension. ${ }^{50}$ This previous study also suggested that the change in line tension is related to the change in $T_{\mathrm{c}}$.

Furthermore, compared with previous studies by Veatch et $a l .{ }^{36}$ and Honerkamp-Smith et al., ${ }^{44}$ the present results do not provide in-depth information about the critical phenomena, such as discussion of the correlation length. Hence, further detailed studies are required.

Finally, we discuss the biological implications of the present study. We demonstrated that $\mathrm{DC} \cdot \mathrm{HCl}$ has the ability to decrease the $T_{\mathrm{c}}$ of multicomponent liposomes. Previously, it has been revealed that changes in the lipid state affects the

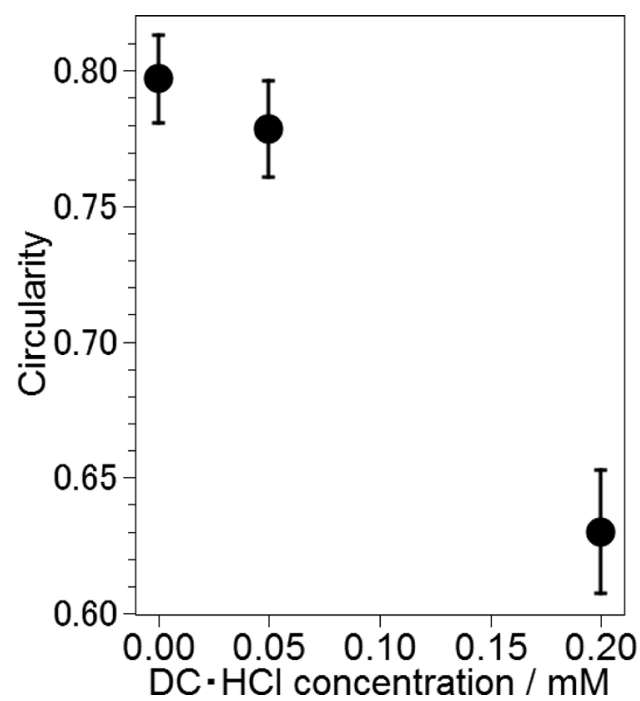

Fig. 5 Circularity of the Lo domains versus $\mathrm{DC} \cdot \mathrm{HCl}$ concentration at $20{ }^{\circ} \mathrm{C}\left(<T_{\mathrm{c}}\right)$. Error bars represent standard errors ( $n \geq 27$ Lo domains under each set of concentrations). 
structure and function of the membrane protein. ${ }^{51,52}$ In terms of the previous and present studies, it was determined that the $\mathrm{DC} \cdot \mathrm{HCl}$ molecules have the ability to influence the function of ion channels by affecting the lipids that surround the ion channels. We expect that these findings will further the fields of pharmacology and medicinal chemistry.

\section{Conclusion}

In summary, we have investigated the influence of $\mathrm{DC} \cdot \mathrm{HCl}$ molecules, a local anaesthetic, on the phase behavior of DOPC $/$ DPPC $/$ Chol $=50 / 25 / 25(\mathrm{~mol} \%)$ liposomes. To confirm these effects, ternary liposomes with $0,0.05$ and $0.2 \mathrm{mM}$ $\mathrm{DC} \cdot \mathrm{HCl}$ were observed at various temperatures and their phase patterns were quantified by angle-averaged 2D-AC functions. As a result, we clarified that the DC.HCl molecules have the ability to change the miscibility transition temperature $\left(T_{\mathrm{c}}\right)$ of ternary liposomes. Furthermore, we calculated the circularity of the Lo domains at $20{ }^{\circ} \mathrm{C}$ to estimate the change in line tension of the Lo/Ld phase boundary, revealing that insertion of the DC molecules into bilayers induces reduction in line tension of the phase boundary with decrease in $T_{\mathrm{c}}$. These results suggest that the DC. $\mathrm{HCl}$ molecules disturb the function of ion channels (anaesthetic function of $\mathrm{DC} \cdot \mathrm{HCl}$ ) by affecting the lipid bilayers that surround the ion channels. Since these physicochemical findings suggest the mechanism of anaesthetic function, this study will play an important role in pharmacology.

\section{Acknowledgements}

The authors thank Dr. Yasuhiro Fujii (Ritsumeikan Univ.), Prof. Tsutomu Hamada (JAIST), Dr. Rina Kagawa (M.D., Univ. Tokyo) and Prof. Miho Yanagisawa (Tokyo Univ. Agr. Tech.) for their significant advises.

\section{References}

1 D. A. Haydon and B. W. Urban, J. Physiol., 1983, 341, 429-439.

2 L. Ebihara, J. E. Hall, R. C. MacDonald, T. J. McIntosh and S. A. Simon, Biophys. J., 1979, 28, 185-196.

3 J. Reyes and R. Latorre, Biophys. J., 1979, 28, 259-280.

4 R. Reigada, J. Phys. Chem. B, 2011, 115, 2527-2535.

5 R. Reigada, PLoS One, 2013, 8, e52631.

6 Y. Muroi and B. Chanda, J. Gen. Physiol., 2009, 133, 1-15.

7 D. A. Hanck, E. Nikitina, M. M. McNulty, H. A. Fozzard, G. M. Lipkind and M. F. Sheets, Circ. Res., 2009, 105, 492-499.

8 T. Yamagishi, W. Xiong, A. Kondratiev, P. Vélez, A. MéndezFitzwilliam, J. R. Balser, E. Marbán and G. F. Tomaselli, Mol. Pharmacol., 2009, 76, 861-871.

9 M. Arcisio-Miranda, Y. Muroi, S. Chowdhury and B. Chanda, J. Gen. Physiol., 2010, 136, 541-554.

10 M. F. Sheets, T. Chen and D. A. Hanck, Eur. J. Appl. Physiol., 2011, 461, 91-97.
11 S. Lee, S. J. Goodchild and C. A. Ahern, J. Gen. Physiol., 2012, 139, 507-516.

12 J. S. Bant, T. K. Aman and I. M. Raman, J. Neurosci., 2013, 33, 4976-4987.

13 T. Hata, H. Matsuki and S. Kaneshina, Colloids Surf., B, 2000, 18, 41-50.

14 T. Hata, T. Sakamoto, H. Matsuki and S. Kaneshina, Colloids Surf., B, 2001, 22, 77-84.

15 H. Matsuki, T. Hata, M. Yamanaka and S. Kaneshina, Colloids Surf., B, 2001, 22, 69-76.

16 K. Takeda, Y. Sano, S. Ichikawa, Y. Hirata, H. Matsuki and S. Kaneshina, J. Oleo Sci., 2009, 58, 369-377.

17 H. Tsuchiya, T. Ueno, M. Mizogami and K. Takakura, Chem.Biol. Interact., 2010, 183, 19-24.

18 K. Simons and J. L. Sampaio, Cold Spring Harbor Perspect. Biol., 2011, 3, a004697.

19 R. Lipowsky and R. Dimova, J. Phys.: Condens. Matter, 2003, 15, S31.

20 H. Tsuchiya, T. Ueno, M. Mizogami and K. Takakura, J. Anesth. Clin. Res., 2010, 24, 639-642.

21 C. Bandeiras, A. P. Serro, K. Luzyanin, A. Fernandes and B. Saramago, Eur. J. Pharm. Sci., 2013, 48, 153-165.

22 T. Baumgart, S. T. Hess and W. W. Webb, Nature, 2003, 425, 821-824.

23 S. L. Veatch and S. L. Keller, Biophys. J., 2003, 85, 3074-3083.

24 A. Diguet, M. Yanagisawa, Y.-J. Liu, E. Brun, S. Abadie, S. Rudiuk and D. Baigl, J. Am. Chem. Soc., 2012, 134, 4898-4904.

25 M. Morita, T. Hamada, Y. Tendo, T. Hata, M. C. Vestergaard and M. Takagi, Soft Matter, 2012, 8, 2816-2819.

26 N. Shimokawa, M. Hishida, H. Seto and K. Yoshikawa, Chem. Phys. Lett., 2010, 496, 59-63.

27 H. Himeno, N. Shimokawa, S. Komura, D. Andelman, T. Hamada and M. Takagi, Soft Matter, 2014, 10, 7959-7967.

28 P. Cicuta, S. L. Keller and S. L. Veatch, J. Phys. Chem. B, 2007, 111, 3328-3331.

29 Y. Sakuma, T. Taniguchi, T. Kawakatsu and M. Imai, Biophys. J., 2013, 105, 2074-2081.

30 D. Chen and M. M. Santore, Langmuir, 2014, 30, 9484-9493.

31 H. S. Muddana, H. H. Chiang and P. J. Butler, Biophys. J., 2012, 102, 489-497.

32 E. Gray, J. Karslake, B. B. Machta and S. L. Veatch, Biophys. J., 2013, 105, 2751-2759.

33 J. Hwang, L. A. Gheber, L. Margolis and M. Edidin, Biophys. J., 1998, 74, 2184-2190.

34 S. Facsko, T. Dekorsy, C. Koerdt, C. Trappe, H. Kurz, A. Vogt and H. L. Hartnagel, Science, 1999, 285, 1551-1553.

35 V. Geringer, M. Liebmann, T. Echtermeyer, S. Runte, M. Schmidt, R. Rückamp, M. C. Lemme and M. Morgenstern, Phys. Rev. Lett., 2009, 102, 076102.

36 S. L. Veatch, P. Cicuta, P. Sengupta, A. Honerkamp-Smith, D. Holowka and B. Baird, ACS Chem. Biol., 2008, 3, 287-293.

37 R. Koynova and M. Caffrey, Biochim. Biophys. Acta, Biomembr., 1998, 1376, 91-145.

38 D. D. Lasic, Biochem. J., 1988, 256, 1-11.

39 K. Yoshida, Y. Fujii and I. Nishio, J. Phys. Chem. B, 2014, 118, 4115-4121. 
40 C. A. Schneider, W. S. Rasband and K. W. Eliceiri, Nat. Methods, 2012, 9, 671-675.

41 S. Chiantia, N. Kahya, J. Ries and P. Schwille, Biophys. J., 2006, 90, 4500-4508.

42 S. L. Veatch, S. S. W. Leung, R. E. W. Hancock and J. L. Thewalt, J. Phys. Chem. B, 2007, 111, 502-504.

43 A. J. García-Sáez, S. Chiantia and P. Schwille, J. Biol. Chem., 2007, 282, 33537-33544.

44 A. R. Honerkamp-Smith, P. Cicuta, M. D. Collins, S. L. Veatch, M. den Nijs, M. Schick and S. L. Keller, Biophys. J., 2008, 95, 236-246.

45 N. Weizenmann, D. Huster and H. A. Scheidt, Biochim. Biophys. Acta, Biomembr., 2012, 1818, 3010-3018.

46 S. A. Akimov, E. A. Hlaponin, P. V. Bashkirov, I. A. Boldyrev, I. I. Mikhalyov, W. G. Telford and I. M. Molotkovskaya,
Biochem. (Mosc.) Suppl. Ser. A: Membr. Cell Biol., 2009, 3, 216-222.

47 J. B. Hutchison, R. M. Weis and A. D. Dinsmore, Langmuir, 2012, 28, 5176-5181.

48 M. Weinrich, H. Nanda, D. L. Worcester, C. F. Majkrzak, B. B. Maranville and S. M. Bezrukov, Langmuir, 2012, 28, 4723-4728.

49 M. Weinrich and D. L. Worcester, J. Phys. Chem. B, 2013, 117, 16141-16147.

50 T. Hamada, R. Sugimoto, T. Nagasaki and M. Takagi, Soft Matter, 2011, 7, 220-224.

51 M. Ø. Jensen and O. G. Mouritsen, Biochim. Biophys. Acta, Biomembr., 2004, 1666, 205-226.

52 A. Laganowsky, E. Reading, T. M. Allison, M. B. Ulmschneider, M. T. Degiacomi, A. J. Baldwin and C. V. Robinson, Nature, 2014, 510, 172-175. 